Musées, Patrimoine et Culture scientifiques et techniques

\title{
Espace pour la vie : un mouvement participatif pour l'engagement citoyen
}

Johanne Landry

\section{OpenEdition \\ Journals}

Édition électronique

URL : http://journals.openedition.org/ocim/1522

DOI : $10.4000 /$ ocim. 1522

ISSN : 2108-646X

Éditeur

OCIM

Édition imprimée

Date de publication : 1 mai 2015

Pagination : $30-37$

ISSN : 0994-1908

Référence électronique

Johanne Landry, «Espace pour la vie : un mouvement participatif pour l'engagement citoyen », La Lettre de I'OCIM [En ligne], 159 | 2015, mis en ligne le 01 mai 2016, consulté le 30 avril 2019. URL: http://journals.openedition.org/ocim/1522 ; DOI : 10.4000/ocim.1522

Ce document a été généré automatiquement le 30 avril 2019.

Tous droits réservés 


\title{
Espace pour la vie : un mouvement participatif pour l'engagement citoyen
}

\author{
Johanne Landry
}

Espace pour la vie, une signature collective composée de quatre symboles représentent les quatre institutions : deux ailes de papillons oranges pour l'Insectarium, deux feuilles vertes pour le Jardin botanique, un manchot bleu pour le Biodôme et deux planètes mauves pour le Planétarium se regroupent et forment une sphère rappelant la planète Terre.
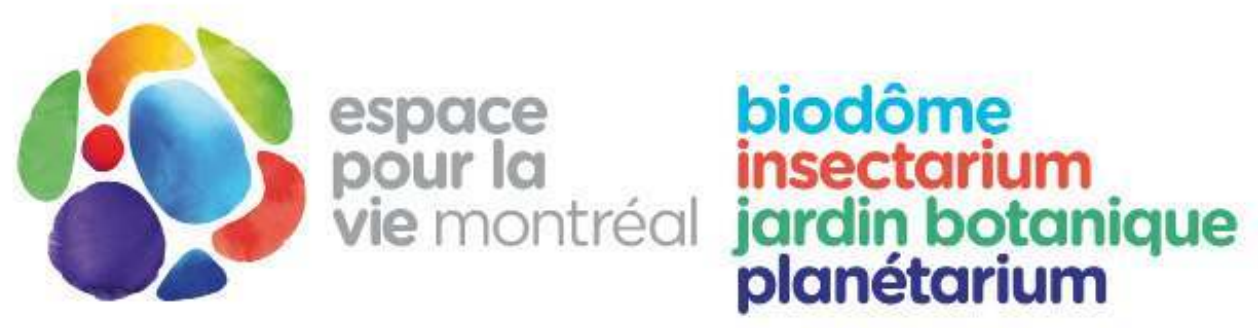

(C) Espace pour la vie

1 Espace pour la vie reçoit près de 1,8 millions de visiteurs chaque année. Ce regroupement d'institutions scientifiques et muséales représente le plus grand complexe en sciences naturelles au Canada. Espace pour la vie est financé principalement par la Ville de Montréal avec une contribution, depuis quelques années, du gouvernement provincial.

2 Depuis leurs créations respectives, le Jardin botanique, le Biodôme, l'Insectarium et le Planétarium ont pour vocation de sensibiliser les visiteurs aux sciences de la nature. Au regard des enjeux auxquels la planète est confrontée, ces quatre institutions ont vu leur rôle évoluer. Au fil des ans, elles ont intégré à leurs discours des informations visant à conscientiser les visiteurs quant à l'impact de leurs gestes sur la nature. Aujourd'hui, l'urgence est telle qu'elles ont décidé d'unir leurs voix et d'initier un vaste mouvement où les citoyens sont invités à inventer une autre façon de vivre en lien avec la nature. Le nouveau logo d'Espace pour la vie vient renforcer la synergie de nos équipes et témoigne 
de l'engagement pour la biodiversité que nous voulons affirmer auprès de la communauté dans notre volonté d'initier un mouvement participatif pour la protection de la nature.

\section{Un peu d'histoire pour comprendre les racines des liens avec la communauté québécoise}

3 La période des années 1930-1970 a permis la mise en place du Jardin botanique, réalisé par le Frère Marie-Victorin, un éducateur très ancré dans sa communauté, avec la collaboration de l'architecte paysagiste américain Henry Teuscher. Dès le début des années 1930, Marie-Victorin convainc le maire de Montréal de construire un jardin avec des arguments politiques: favoriser le rapprochement des Canadiens-Français avec la science et la recherche universitaire; donner des emplois aux Québécois venus des campagnes pour trouver du travail en ville et pour les rapprocher de la nature qu'ils ont quittée pour venir dans un milieu urbain; donner aux enfants une école de l'éveil et un lieu pour apprendre à jardiner... Suite au décès accidentel de Marie-Victorin, l'ethnobotaniste Jacques Rousseau prend la direction du Jardin botanique et développe des liens étroits avec la communauté des Premières Nations et des Inuits afin de comprendre les liens qui les unissent à la nature. La voie était tracée...

\section{Les années 1970-1990, une période effervescente pour le verdissement et l'ouverture sur le monde}

4 Au début des années 1980, les quatre institutions (le Jardin botanique, l'Aquarium de Montréal, le Parc Angrignon et le Planétarium Dow) sont regroupées sous le vocable Fonds des équipements scientifiques qui fait partie du Service des Parcs de la Ville de Montréal. Pierre Bourque est alors directeur du Jardin botanique et du Service des Parcs et des Espaces verts de la Ville de Montréal.

Divers projets initiés dès les années 1980 en font une ville verte, une «ville de la douceur $»^{1}$ : on voit apparaître les pistes cyclables et les projets de verdissements. Le projet Place au soleil a permis à de nombreux citoyens d'aménager des espaces de vie pour remplacer des ruelles bétonnées non adaptées aux jeux des enfants. Il s'est développé par la suite, au sein de la ville, un véritable réseau de ruelles vertes (http://ruelleverte.com/) qui se transforment parfois en ruelles blanches l'hiver (http://aduq.ca). Le réseau des pistes cyclables et la multiplication des espaces verts viennent offrir aux citoyens des projets novateurs qui, aujourd'hui, ont une répercussion importante pour améliorer la vie de la communauté montréalaise. 


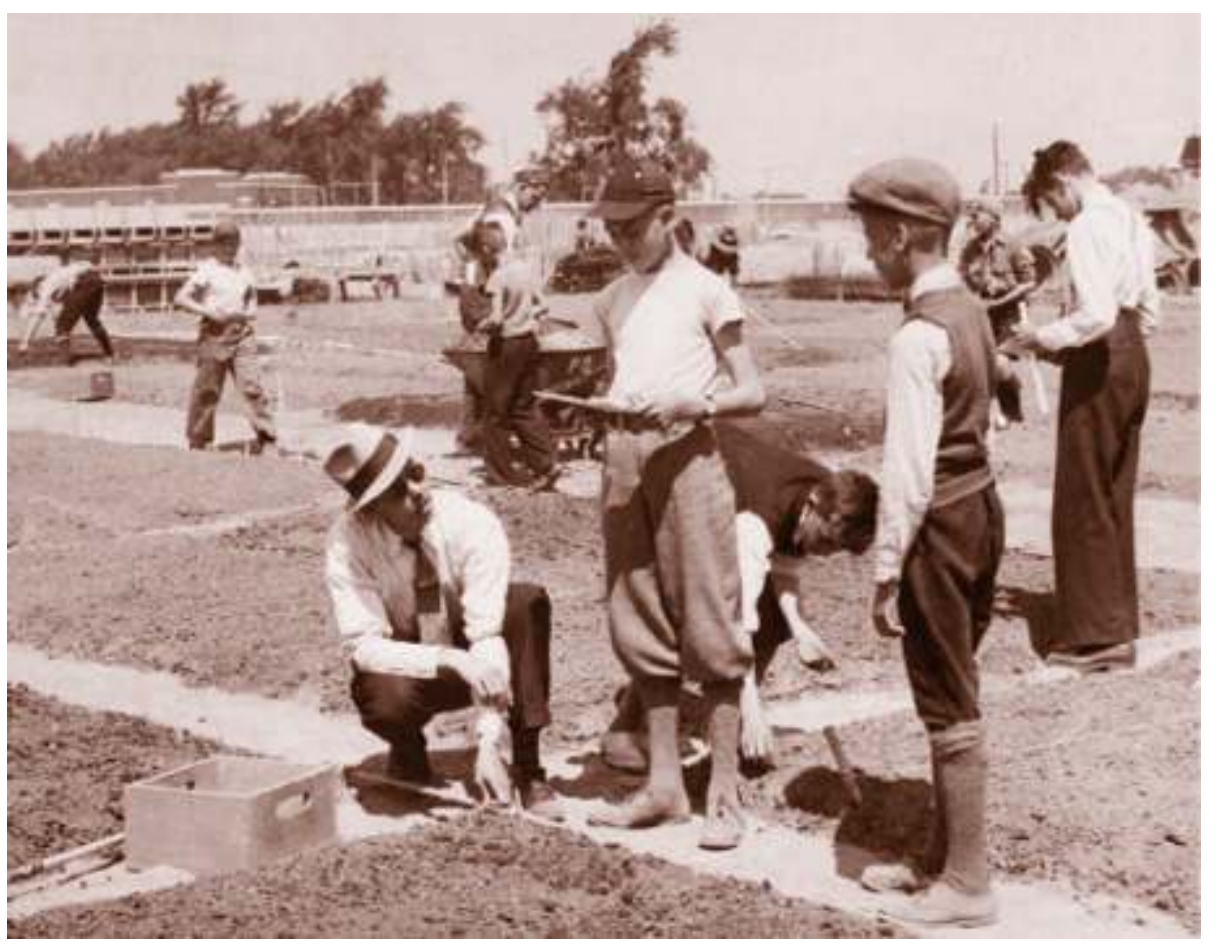

(C) Jardin botanique de Montréal

6 C'est aussi à cette époque que la Ville continue de s'ouvrir sur le monde, ouverture initiée avec l'Exposition universelle de 1967 et poursuivie avec les Jeux Olympiques de 1976. En 1980, la Ville propose d'organiser les Floralies internationnales dans l'ancien Vélodrome olympique : à cette occasion, la Chine et le Japon offrent de magnifiques collections de penjings et de bonsaïs au Jardin botanique. Suite à ces dons, la construction du Jardin et du pavillon japonais ainsi que celle du Jardin de Chine se concrétisent respectivement en 1989 et 1991. L'équipe constate également que le Vélodrome, avec son toit de lanterneaux transparents, offre des possibilités pour relocaliser les collections animales de l'Aquarium et du parc Angrigon qui nécessitent rapidement une rénovation. C'est alors que le directeur propose de recycler le Vélodrôme en Biodôme qui ouvrira en 1992. Cette réutilisation d'un bâtiment représentait déjà un premier geste vers une démarche de Développement durable.

7 Cet événement des Floralies a vraiment donné le coup d'envoi de l'engouement des Québécois pour l'horticulture ornementale et le verdissement des quartiers. On le constate par la multiplication des chroniques horticoles dans les médias, presse, radio et télévision à cette époque. La pratique horticole devient rapidement le loisir préféré des Québécois pendant de nombreuses années.

8 Au même moment, on voit apparaître dans l'équipe de recherche du Jardin botanique, un groupe qui se spécialise dans la phytotechnologie de filtration de l'eau par les plantes. Au début des années 1990, cette équipe de recherche participe au projet de la Biosphère et de la plage de l'île Sainte-Hélène pour réaliser des marais filtrés par les plantes. 


\section{L'agriculture urbaine, d'hier à aujourd'hui}

D'autres projets novateurs ont vu le jour au sein du Jardin botanique Ces projets ont ensuite fait place à des mouvements citoyens qui ont pris la relève, notamment pour le développement de l'agriculture urbaine à Montréal.

Créés en 1938 par le Frère Marie-Victorin, les Jardins-jeunes font aujourd'hui partie d'un vaste mouvement d'agriculture urbaine qui prend de plus en plus d'ampleur au niveau planétaire. Par cette activité éducative et pédagogique, les enfants réalisent que la nourriture vient de la terre... cette terre nourricière qui offre aux familles une source d'alimentation constante, en liaison avec les saisons.

Les Jardins-jeunes ont pour mission de favoriser l'apprentissage de l'agriculture urbaine auprès des jeunes enfants qui viennent jardiner leur lopin de terre individuel de mai à septembre au Jardin botanique de Montréal.

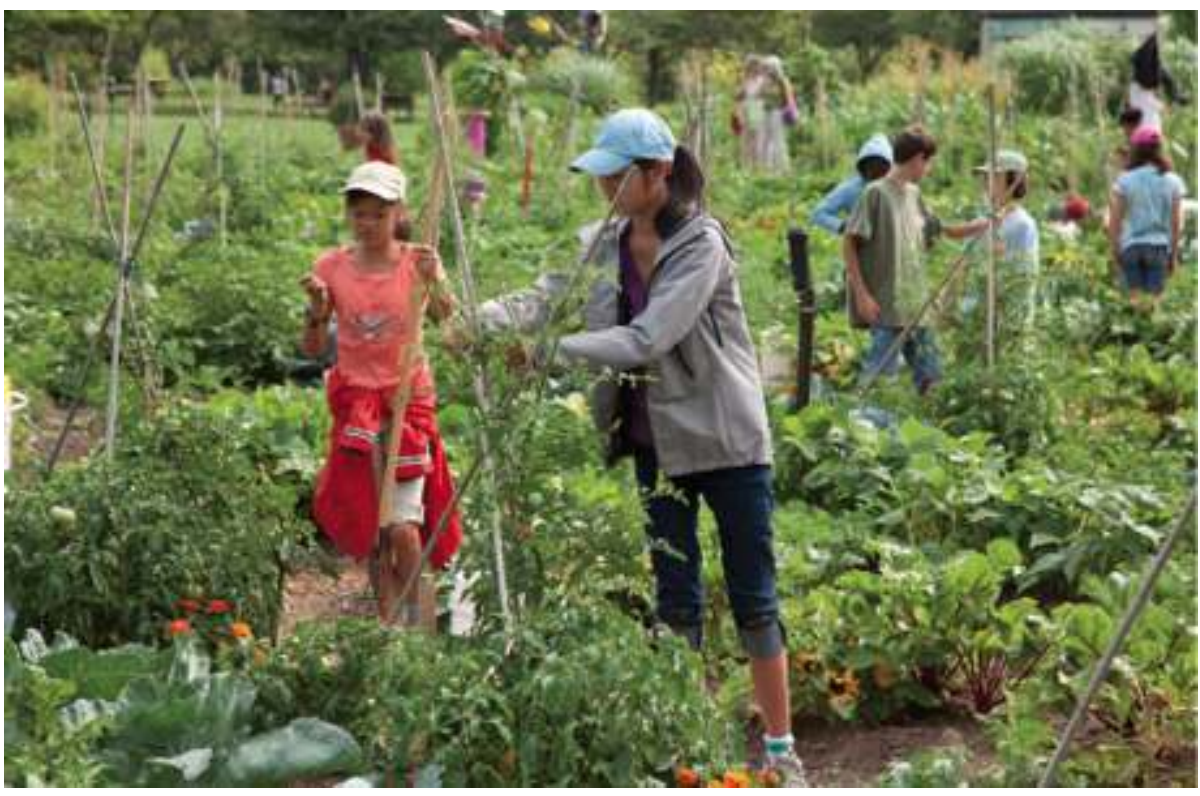

(c) Jardin botanique de Montréal/Michel Tremblay

En 2013, un projet de recherche, dirigé par Irène Rahm, professeur à l'université de Montréal, permet d'analyser les intentions des jeunes qui participent à ce programme d'agriculture urbaine au Jardin botanique. Un élément intéressant qui ressort de ces entrevues est la fierté des jeunes qui ramènent des légumes à la maison et qui, par leur travail, permettent de nourrir la famille à faible coût... ${ }^{2}$

Dans les années 1970, des citoyens moins bien nantis de Montréal, demandent au Jardin botanique où jardiner des légumes afin de nourrir leurs familles. Le premier jardin communautaire de Montréal voit le jour en 1972. En plus de cette initiative citoyenne inaugurée il y a 43 ans, on voit apparaître également des jardins collectifs (non divisés en lot individuels) et un mouvement citoyen d'agriculture urbaine partout dans la ville. Depuis 7 ans, l'UQAM propose une école d'agriculture urbaine chaque été au mois d'août ( http://ecoleagricultureurbaine.org/).

Le Jardin botanique a aussi géré pendant plusieurs années une ferme, la Ferme Angrignon, afin d'offrir aux jeunes Montréalais de l'ouest de la ville une occasion de découvrir les 
animaux de ferme et les jardins potagers. Fermée depuis 2008, cette ferme sera réouverte dans le cadre d'un projet majeur de développerment d'un pôle d'agriculture urbaine pour 2018.

\section{Comment aider les citoyens à cultiver la terre et à protéger la biodiversité ?}

Depuis près de quinze ans, le Jardin botanique organise, à la fin du mois de mai, les Rendez-vous horticoles, une grande foire horticole qui accueille plus de 100 exposants (producteurs de plantes, associations d'agriculture urbaine...). Près de 18000 visiteurs viennent y faire leurs achats et discuter avec les spécialistes du Jardin pour améliorer leurs pratiques de jardinage afin de favoriser la protection de la biodiversité. Le Jardin botanique de Montréal accueille également dans ses locaux, l'École des métiers de l'horticulture de la Commission scolaire de Montréal, pour participer à préparer la relève dans le domaine de l'horticulture,

Afin de répondre aux besoins des citoyens, Espace pour la vie offre gratuitement plusieurs services à la communauté montréalaise. Depuis plus de 25 ans, des botanistes chevronnés animent le comptoir de renseignements horticoles situé au Complexe d'accueil du Jardin botanique : ils prodiguent des conseils aux citoyens qui viennent les rencontrer pour faire identifier des plantes, discuter de problèmes de ravageur ou de maladie ou simplement pour recevoir des conseils sur la culture et l'entretien écologique des végétaux. Ces botanistes sont responsables de la mise à jour du carnet horticole du site Internet du Jardin botanique pour répondre aux questions des nombreux jardiniers amateurs au Québec et dans le monde (http://espacepourlavie.ca/carnet-horticole-et-botanique). Un service similaire par courriel est offert par les entomologistes de l'Insectarium pour répondre aux questions des citoyens qui veulent faire identifier des insectes observés dans leur environnement (http://espacepourlavie.ca). Développé en 2013 en collaboration avec le regroupement des Éco-quartiers de Montréal, le programme participatif Mon jardin Espace pour la vie, invite les citoyens à aménager chez eux, un espace dédié aux oiseaux, aux papillons ou simplement pour la biodiversité.

\section{Le Développement durable et l'inclusion sociale}

Dans les années 1990, Espace pour la vie se questionne sur les enjeux du Développement durable non seulement dans les aspects physiques de ses opérations mais aussi pour son implication sociale dans la communauté. Dès la construction du Biodôme, un comité accessibilité est créé afin de faciliter l'inclusion de toutes les personnes. Depuis 1994, le programme PAVÉ (Programme Annuel de Visites Éducatives), offre des activités pour enfants ayant des déficiences physiques ou intellectuelles. Le Biodôme organise aussi de 1994 à 2011 le projet Déchets d'œuvres, un concours international de jouets fabriqués à partir de matériaux recyclés développés avec le club Deux tiers. Ce projet éducatif en arts plastiques aborde des thèmes comme l'économie, la surconsommation, les inégalités entre les pays du Sud et du Nord et les différences et ressemblances culturelles. En 1999, le Jardin botanique inaugure la Cour des sens, un jardin thématique qui offre une variété d'activités pour les personnes ayant des déficiences visuelles mais aussi pour les visiteurs avec des capacités physiques limitées. 
Les trottoirs jardins : depuis 4 ans, à l'initiative de Richer Beaulieu, 1000 tonnes d'asphalte ont été remplacées par des jardins de rue aménagés et entretenus par les habitants du quartier.

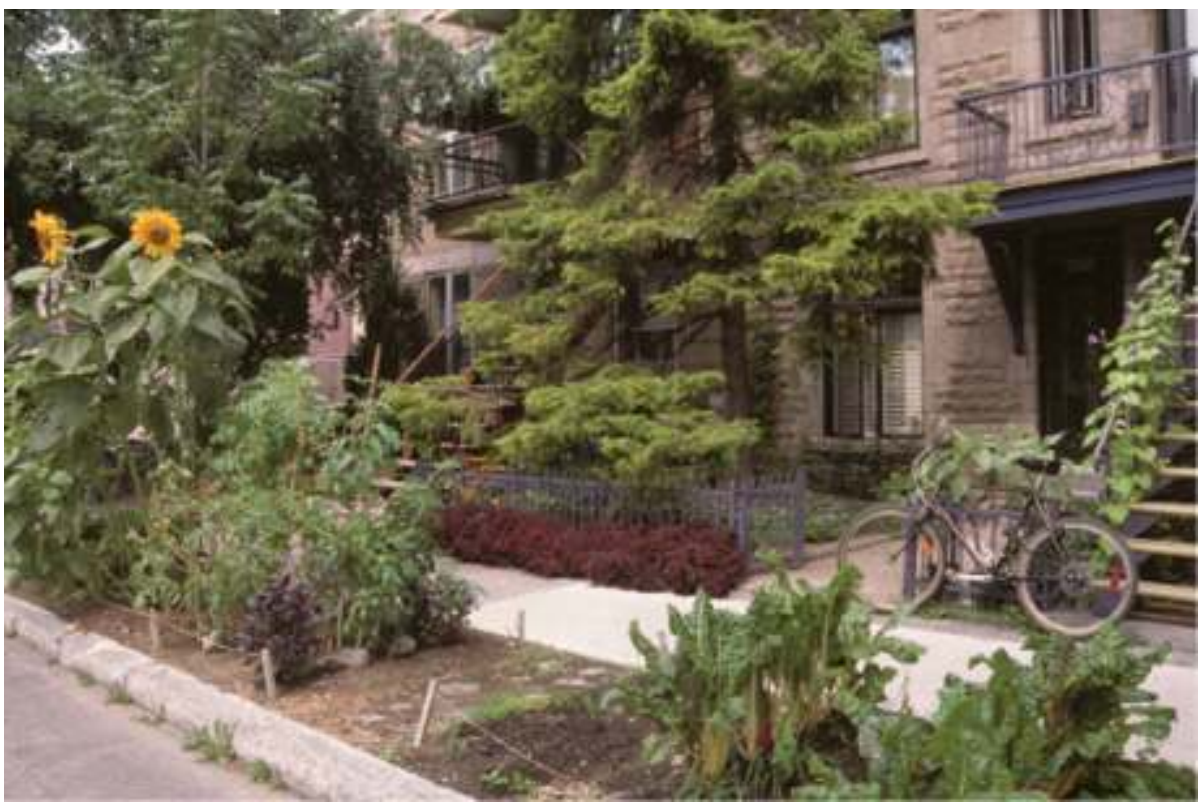

(c) Jardin botanique de Montréal/Lise Servant

Cependant, peu de ressources sont disponibles pour déveloper une politique formelle qui guiderait le Développement durable d'Espace pour la vie. Grâce au financement de l'Accord Canada-France, Espace pour la vie initie une réflexion sur «Musées et Développement durable» (2007-2009) avec le Muséum national d'Histoire naturelle de Paris (MNHN). En 2010, un conseiller en Développement durable, engagé au sein de l'équipe d'Espace pour la vie, présente quelques mois plus tard sa Politique de Développement durable (http://espacepourlavie.ca/politique-de-developpement-durable ) en lien avec le Plan d'action Développement durable de la Ville de Montréal. À cette époque, l'équipe des services techniques développe un projet d'ingénierie, incluant la géothermie, permettant à Espace pour la vie de faire un geste concret pour l'économie d'énergie à la fois au Biodôme et à l'Insectarium de Montréal.

En 2010, le Jardin botanique inaugure son Parcours Kéroul d'accessbilité universelle, un parcours de 2,55 km spécialement aménagé et adapté pour les personnes à mobilité réduite. Récemment, Espace pour la vie a fait un pas de plus dans la démarche de Développement durable en développant le Projet Sol un des plus importants projets d'économie sociale au Québec. Depuis novembre 2013, Espace pour la vie a fait appel à plusieurs entreprises d'insertion socioprofessionnelles en services alimentaires afin de renouveler son offre alimentaire au Biodôme et au Planétarium Rio Tinto Alcan. Un menu à prédominance végétarienne et locale, de certification biologique ou écologique qui favorise le développement de la collectivité en favorisant la réinsertion sociale. Un geste concret et efficace pour l'environnement naturel et humain. 


\section{L'importance des sciences citoyennes à Espace pour la vie} en créant le programme Monarque sans frontière qui allie recherche et éducation. Ce projet vise à sensibiliser le public (et plus particulièrement les enfants) à la beauté et à la fragilité de la nature tout en lui faisant réaliser qu'il a le pouvoir de changer les choses. Les monarques étiquetés retrouvés le long de leur voie migratoire ou sur les sites d'hivernage fournissent des données importantes pour le programme de recherche «Monarch Watch » de l'université du Kansas. Un projet de recherche est actuellement en préparation à l'Insectarium pour analyser la disparition des habitats du monarque en Amérique du Nord. Le responsable de la recherche et des collections à l'Insectarium, Maxime Larrivée, est aussi fondateur et directeur du projet E-butterfly, un site de sciences citoyennes qui permet de faire l'inventaire des papillons au Canada grâce à l'apport de bénévoles amateurs.

La Forêt éphémère est entièrement composée de près de 1000 sapins de Noël récupérés après la période des fêtes pour envoyer un message collectif sur la protection de la biodiversité.

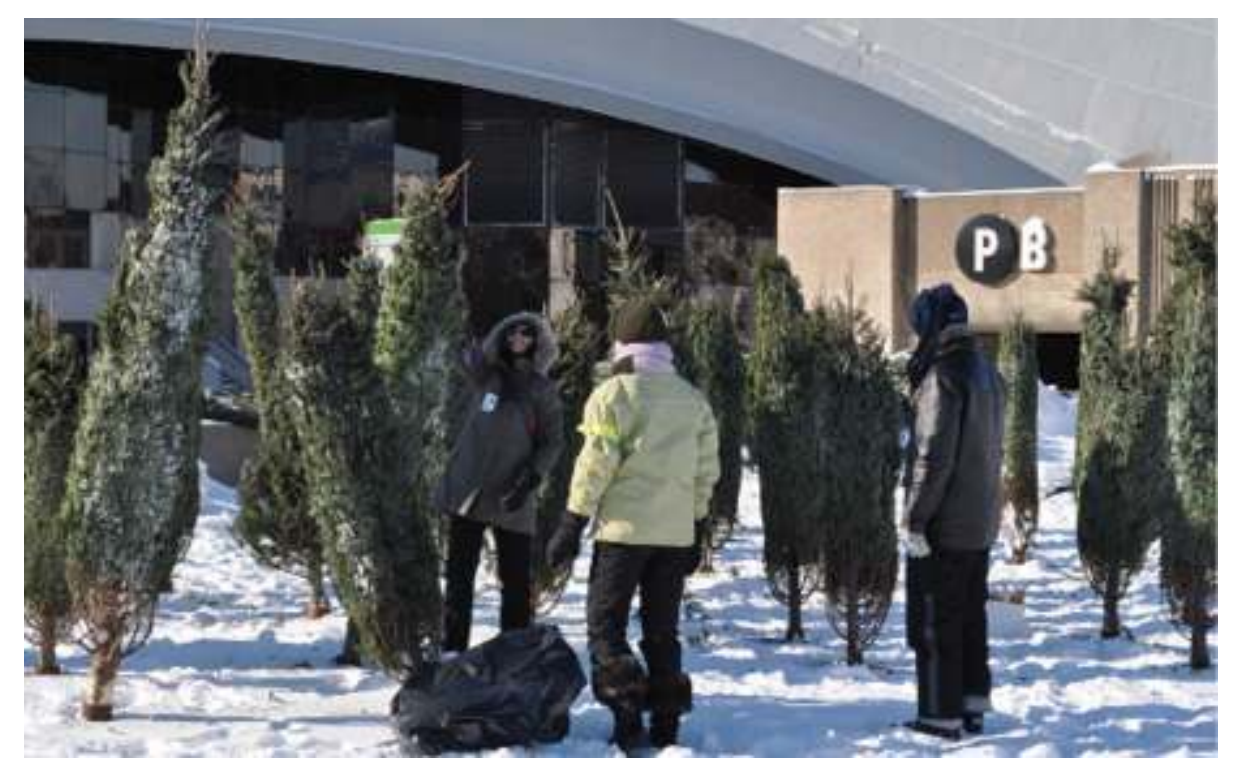

(c) Espace pour la vie

Initiée par Anne Breault, une chercheur du Biodôme en 1999, le projet Sem'ail est un programme de sensibilisation et d'éducation pour l'ail des bois, une espèce vulnérable au Québec, afin de promouvoir sa protection et favoriser sa restauration. De 2000 à 2004, près d'un million de graines d'ail des bois ont été distribuées à des propriétaires d'érablières intéressés par la sauvegarde de l'espèce. De plus, 444000 bulbes saisis 
(provenant de cueillettes illégales) par les agents de la faune ont été replantés par des participants de Sem'ail. Depuis 2010, l'équipe du Biodôme coordonne Sem'ailjr, un volet éducatif destiné au milieu scolaire.

\section{Un positionnement affirmé pour une participation citoyenne}

La participation citoyenne ne date pas d'hier à Espace pour la vie. Dès 1985, l'équipe des programmes publics du Jardin botanique organise l'événement Le Grand bal des citrouilles qui célèbrera son trentième anniversaire en octobre 2015. Les citoyens, que ce soit en famille ou en groupe scolaire, décorent des citrouilles selon une thématique annuelle afin de participer au concours et contribuer à réaliser une exposition dans la grande serre du Jardin botanique.

30 ans de participation citoyenne : depuis 1985 , les familles et les écoles montréalaises participent au concours du Grand bal des citrouilles, en décorant ces cucurbitacées qui sont ensuite exposées dans la Grande serre du Jardin botanique...

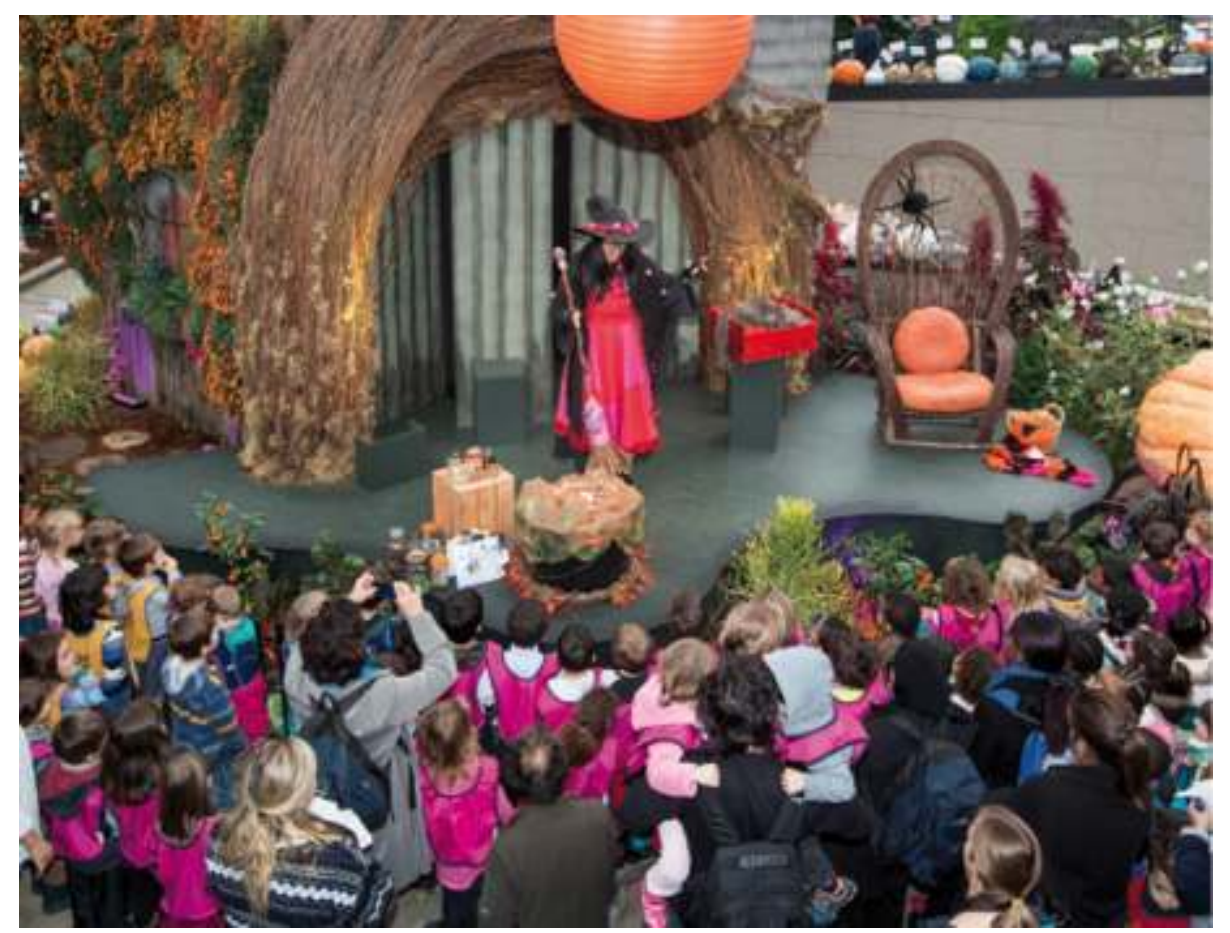

(C) Jardin botanique de Montréal/Claude Lafond

Plus récemment en 2010, dans le cadre de l'année de la biodiversité, Espace pour la vie organise l'événement Les balles de vie, en collaboration avec des jeunes des écoles du Québec. Plus de 4200 enfants participent à cette journée afin d'ensemencer et fleurir le chemin entre le Biodôme et l'Insectarium.

La Forêt éphémère est une œuvre temporaire, érigée conjointement avec les Montréalais, aux abords du Biodôme et du Stade olympique. En 2013, la Forêt éphémère a été reprise par les citoyens montréalais dans un espace intitulé «Le champ des possibles» dans un des arrondissements de la Ville de Montréal. Le mouvement se poursuit avec l'aide de citoyens bénévoles qui veulent s'engager pour la biodiversité. 
L'exposition 1000 jours pour la planète au Centre sur la biodiversité présente des gestes concrets faits par les citoyens pour la préservation de la biodiversité et entre autre une œuvre de Chloé B. Fortin conçue avec les bouchons de plastique amenés par les Montréalais et qui symbolise ce qu'on appelle les « continents de déchets ».

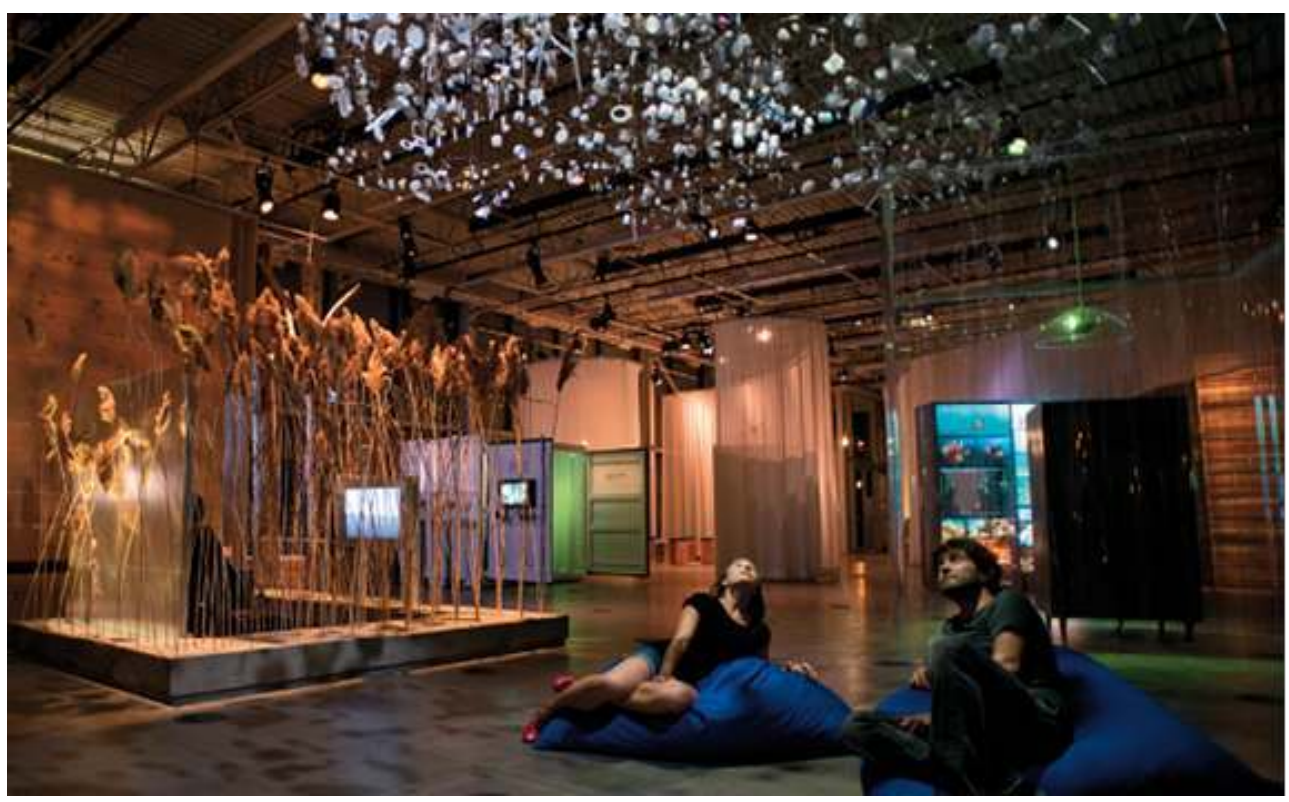

(C) Barry-Russell

En 2012, suite à une consultation publique avec la communauté (citoyens, partenaires, employés...) animée par l'Institut du Nouveau Monde (INM), Espace pour la vie développe une Charte de Participation citoyenne pour affirmer sa volonté de travailler étroitement avec la communauté montréalaise (http://espacepourlavie.ca). Cet engagement s'est concrétisé également tout au long du processus de développement des grands projets d'Espace pour la vie. Le Centre sur la biodiversité, situé au Jardin botanique, est un centre de recherche et de conservation des collections botaniques et entomologiques qui a été inauguré en juin 2012. Dans le cadre de la première exposition 1000 jours pour la Planète, une volonté s'affirme afin de promouvoir également les gestes concrets faits par les citoyens pour la préservation de la biodiversité. Le Programme des ambassadeurs présente des initiatives de citoyens qui soit font des gestes au quotidien ou qui démarre des projets collectifs de programmes d'échanges de vêtements d'enfants, d'aménagements de cours d'écoles ou de jardins sur les trottoirs de la ville. Comme Robert Janes le dit dans son ouvrage, les musées doivent répondre aux enjeux de la société et permettre de construire un futur soutenable ${ }^{4}$. 
Les Arts s'invitent au jardin : depuis 2014, le Jardin botanique offre, les dimanches à ses visiteurs, une programmation musicale pour leur permettre de redécouvrir la floraison saisonnière de ses jardins thématiques.

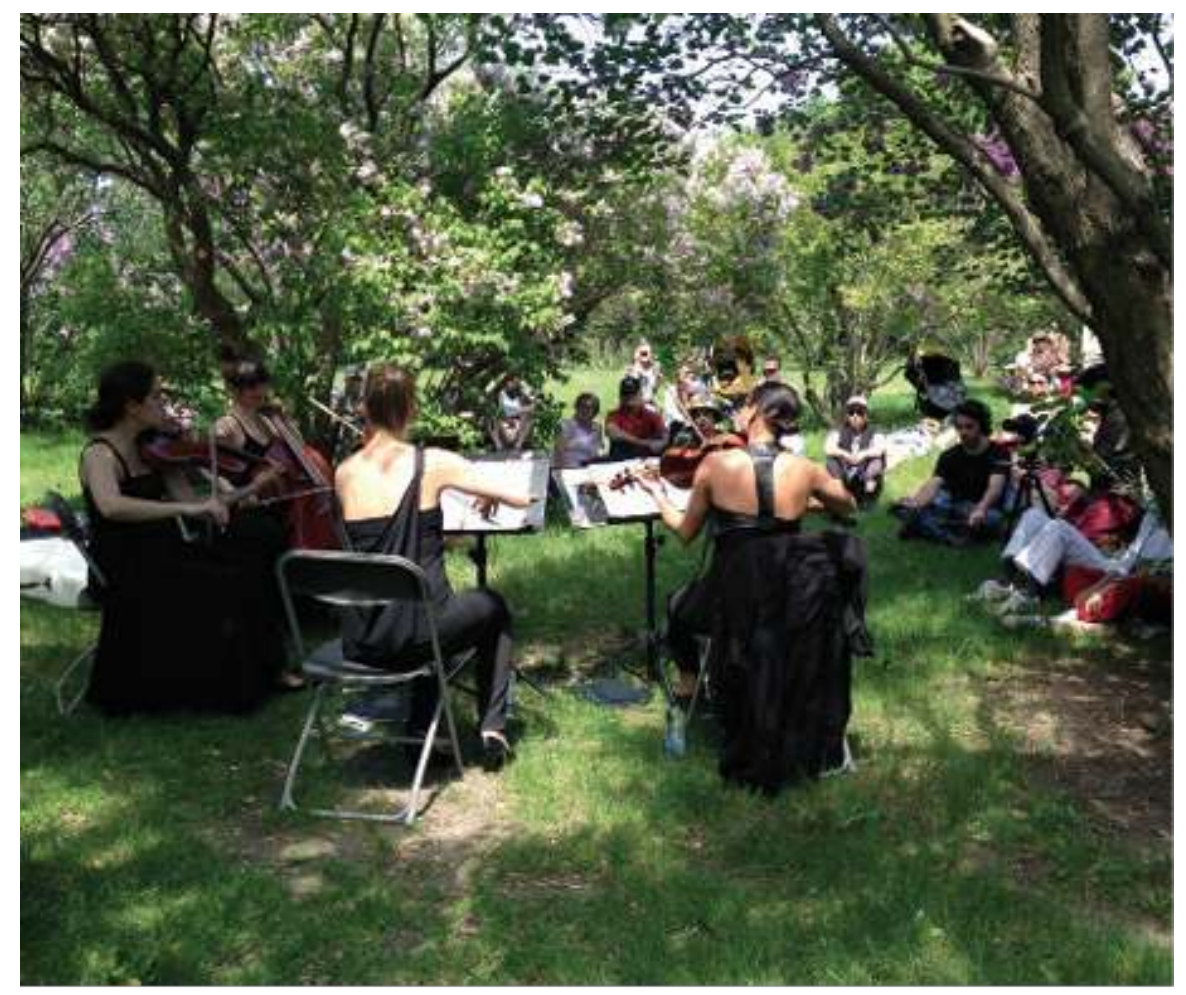

(C) Johanne Landry

\section{Les années thématiques pour dialoguer plus efficacement avec la communauté}

Depuis 2014, Espace pour la vie développe une thématique commune pour les quatre institutions afin de simplifier la programmation annuelle pour ses publics. Cette approche thématique permet aux visiteurs de comprendre plus facilement l'offre multiple d'Espace pour la vie et de profiter des moments de contemplation et de délectation proposés par les institutions.

L'année 2014 s'est déroulée sous le thème de la lenteur, Dépêchez-vous de ralentir. Au Biodôme, un espace dédié aux paresseux et un spectacle pour les familles ont été développés. Au Planétarium, un spectacle sur le temps permet de comprendre la relativité du temps de l'univers et des planètes. À l'Insectarium, un espace dédié au chant des insectes accompagné de musiciens a permis aux visiteurs de se rapprocher du monde des insectes. Au Jardin botanique, une programmation artistique Les Arts s'invitent au Jardin a été offerte aux visiteurs qui se sont prélassés sur des chaises Adirondak réparties dans les jardins en fleurs afin de se relaxer et d'améliorer leur qualité de vie. Une étude récente a démontré que les visiteurs viennent dans les jardins botaniques non seulement pour apprendre et avoir des activités de loisirs en familles mais aussi pour enrayer les effets stressants de la vie urbaine ${ }^{5}$. 
L'année 2015 est celle de la Lumière avec un nouveau spectacle au Planétarium et une installation artistique de Lucioles à l'Insectarium. Les Jardins de lumière reviennent à l'automne au Jardin botanique pour proposer une expérience de célébration de la lumière au Jardin de Chine et au Jardin japonais.

La thématique de l'année 2016 sera importante puisqu'elle sera consacrée au lien EnfantNature. En effet, il est essentiel de toucher les visiteurs dès leur très jeune âge. Richard Louv contate les effets néfastes de ce déficit nature chez les jeunes enfants, fascinés de plus en plus par les jeux électroniques plutôt que par la découverte de la nature autour d'eux ${ }^{6}$.

\section{Un chantier animation : un engagement au service des générations futures}

30 À l'automne 2014, l'équipe de programmation du Jardin botanique a mené une réflexion collective sur sa vision d'avenir et les enjeux sociétaux pour mieux répondre aux besoins de nos futurs visiteurs. Quatre axes stratégiques ont été définis pour les prochaines décennies : les Premiers contacts (pour favoriser, dès le jeune âge, une conscience intime de soi dans la nature), l'Agriculture urbaine (pour améliorer la santé humaine), le lien Natureculture (pour favoriser le mieux vivre ensemble et l'ouverture sur l'altérité), les Clés de découverte (pour favoriser l'engagement de citoyens informés pour l'environnement). Un défi de taille certes, mais essentiel pour assurer l'avenir de nos petits-enfants et la pérennité de cette planète.

\section{Un engagement éthique s'affirme pour la biosphère}

Grâce à un troisième Accord Canada-France (2012-2014), Espace pour la vie a collaboré avec le MNHN et les musées de la Civilisation de Québec afin de développer une déclaration commune sur l'Éthique de la biosphère. Ce travail de co-création avec le personnel des 3 institutions participantes a été présenté aux médias et aux partenaires en décembre $2014^{7}$. Cette déclaration vient guider nos personnels afin d'être encore plus conscient de notre impact sur la biosphère et de poursuivre le travail de sensibilisation de nos publics au développement soutenable de notre planète. 
Les jeunes Montréalais dans leurs jardins.

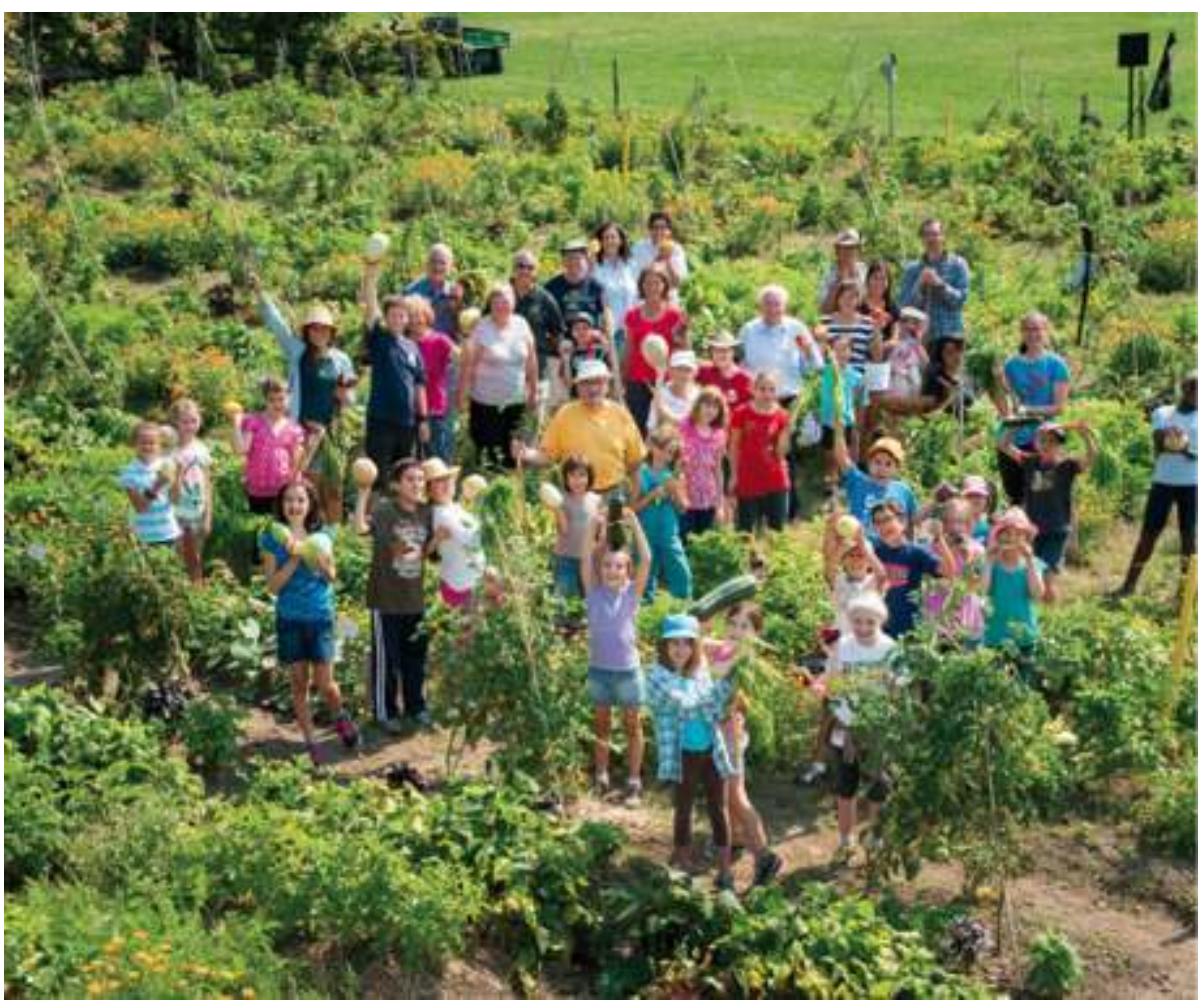

(c) Jardin botanique de Montréal

Devant la situation fragile de la biosphère, que ce soient les changements climatiques ou la disparition de la biodiversité, les muséums et les jardins botaniques ont une responsabilité sociale pour conscientiser nos publics à l'urgence de la situation. Travailler avec les citoyens pour créer des milieux propices à la vie. Les humains ont une curiosité naturelle pour le vivant : c'est ce que le célèbre entomologiste américain Edgar 0 . Wilson a décrit en proposant le terme de biophilie ${ }^{8}$ Nous sommes attirés par le vivant, que ce soient les fourmis qui se promènent le long d'une branche, les outardes qui volent dans le ciel ou les arbres qui se balancent dans le vent. Aider nos visiteurs à retrouver leur véritable nature humaine et comprendre notre interdépendance avec les autres êtres vivants, c'est l'engagement qu'Espace pour la vie veut poursuivre avec la communauté afin de reconnecter l'humain avec la nature.

\section{L'agriculture urbaine, un enjeu important pour les Montréalais}

Depuis 2011, le Droit d'initiative de consultation publique permet aux citoyens d'obtenir, par le dépôt d'une pétition, la tenue de consultation publique sur des projets, des idées ou des causes qui leur sont chers. Résultat de la Charte montréalaise des droits et responsabilités, ce droit permet aux citoyens de contribuer de façon positive au développement de la ville ou de leur arrondissement.

34 En 2011, le droit d'initiative a été utilisé pour la première fois par le Groupe de travail en agriculture urbaine. Plus de 29000 signatures ont été recueillies alors qu'il n'en fallait que 
15000 pour un sujet relevant de la Ville centrale. L'Office de consultation publique de Montréal a tenu une consultation publique sur le sujet au printemps 2012 et a rendu public son rapport le 3 octobre 2012. À la suite de cette consultation, la Ville crée un Comité de travail de la collectivité montréalaise sur l'agriculture urbaine avec la participation de la société civile afin de développer les meilleures pratiques dans ce domaine.

En 2013, dans les divers arrondissements de Montréal, on compte 96 jardins communautaires qui accueillent chacun 75 à 100 familles.

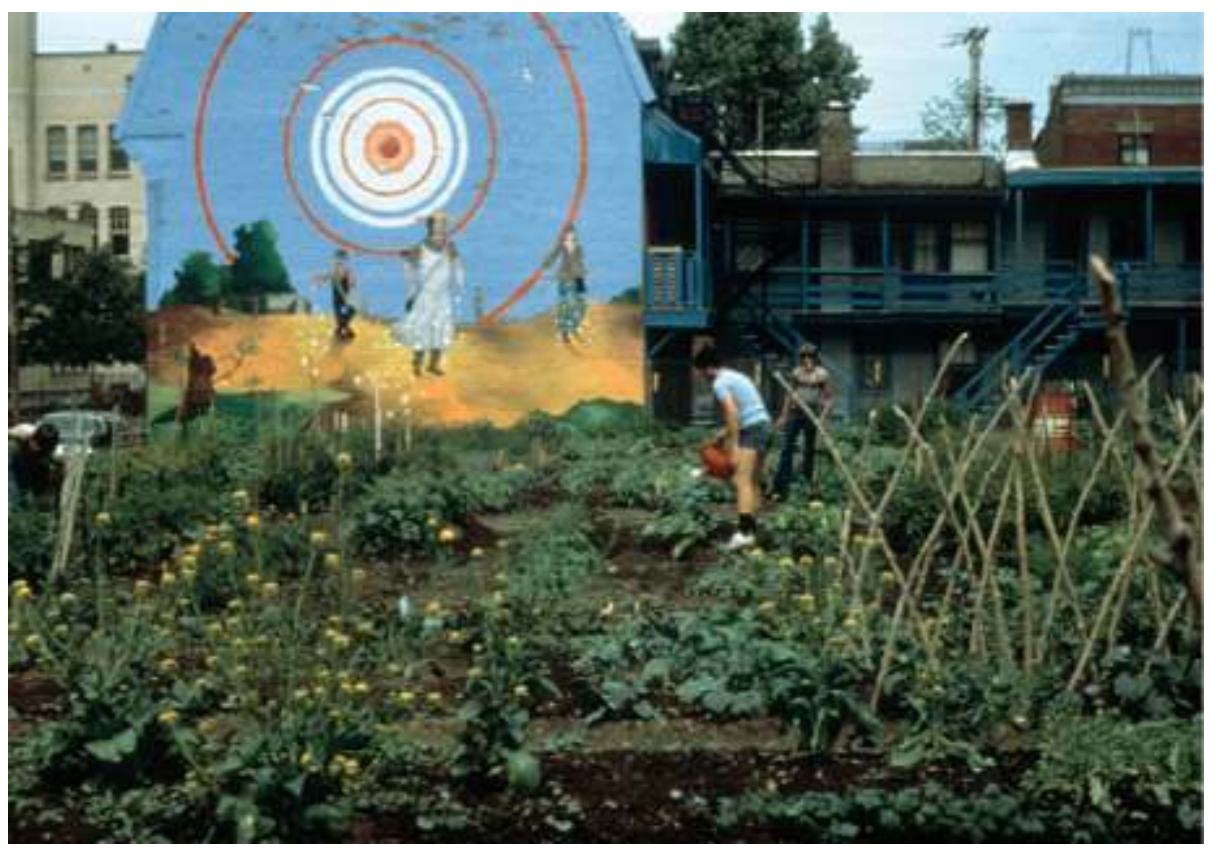

(c) Jardin botanique de Montréal Développement durable est issue du Sommet de Montréal tenu en juin 2002. En octobre 2003, le maire signe la Déclaration de principe de la collectivité montréalaise en matière de Développement durable (http://ville.montreal.qc.ca). Fruit de la collaboration de plus de 180 organismes de tous les horizons de la société montréalaise, le Plan de Développement durable de la collectivité montréalaise 2010-2015 fait suite au premier plan stratégique qui couvrait la période 2005-2009.

En 2006, le gouvernement du Québec adopte la Loi sur le Développement durable qui consacre un véritable choix de société en faveur du Développement durable. 
Conformément à cette loi, le ministère de la Culture, des Communications et de la Condition féminine (MCCCF) rend public, en avril 2009, son plan d'action en matière de Développement durable. L'élaboration d'un Agenda 21 de la Culture du Québec constitue l'action phare de ce plan d'action et s'est amorcée le 20 septembre 2010 ( www.agenda21c.gouv.qc.ca). En 2014, le gouvernement québécois publie un document intitulé: Stratégie gouvernementale de développement durable révisée 2015-2020 où il réitère l'intégration de la culture au Développement durable (www.mddelcc.gouv.qc.ca).

\section{Les grands projets de développement sous le signe du Développement durable, de la participation citoyenne et de la co-création}

39 Le nouveau Planétarium Rio Tinto Alcan a ouvert ses portes en avril 2013 suite à un concours international d'architecture. Il a remporté la certification platine dans la catégorie Leed pour sa réalisation exceptionnelle répondant à la fois aux exigences environnementales (énergie, matériaux...) et celles de l'inclusion sociale pour l'accessibilité physique. Ce nouveau planétarium innove par sa qualité architecturale mais aussi par l'audace de son approche conceptuelle. La réalisation de deux salles de spectacles différentes peut contrarier certains puristes mais l'apport des artistes a définitivement contribué au renouvellement de la programmation. Le " Théâtre de la voie lactée » est dédié aux spectacles scientifiques avec ses nouveaux équipements à la pointe de la technologie, toujours animé en direct par un astronome. Le second, le "Théâtre du chaos ", présente des spectacles artistiques innovants qui surprennent les visiteurs qui s'attendent plutôt à des présentations scientifiques traditionnelles. Cette approche de programmation inclusive donne la possibilité à la communauté artistique québécoise de s'inspirer de thématiques scientifiques pour présenter des spectacles artistiques innovants dans un nouvel espace audio-visuel.

40 Suite à ce premier concours international d'architectural, Espace pour la vie a décidé de poursuivre cette démarche avec le Bureau de design de Montréal pour les trois projets majeurs de développement soit, la Migration du Biodôme, la Métamorphose de l'Insectarium et le Pavillon de verre du Jardin botanique. Au début de 2014, Espace pour la vie a tenu des ateliers créatifs avec des citoyens afin d'enrichir, d'affiner et de valider des idées de concepts pour ces trois projets d'envergure :

41 - le renouvellement du Biodôme, sur le thème de la Migration, s'est appuyé sur une démarche participative avec les employés du Biodôme et des partenaires extérieurs qui sont venus enrichir la vision de développement de cette institution. Le visiteurs parcoureront les quatre écosystèmes selon un tout nouveau cheminement qui leur permettra de voir l'espace sous la verrière immense de cet ancien Vélodrome. Un réseau de passerelles aériennes et un café surplombant l'écosystème subpolaire agrandi complètent ce nouvel ensemble muséal. L'inauguration du projet du Biodôme viendra clôturer les cérémonies du 375e anniversaire de Montréal en décembre 2017.

- ouvert en 1990, l'Insectarium prépare, depuis deux ans, sa Métamorphose avec l'aide de la communauté montréalaise, dans le cadre d'un processus de co-création unique et inspirant. Un living lab et des ateliers créatifs avec des scientifiques, des artistes, des architectes et des communicateurs ont permis le développement d'un concept innovant qui a servi de base pour le concours architectural. Une proposition à la fois audacieuse et 
sensible qui prévoit la création de lieux transparents qui dévoilent l'envers du décor et d'espaces immersifs propices à des rencontres authentiques entre les humains et les insectes. Le projet Métamorphose ouvrira ses portes au public en 2019.

- l'équipe de conception du Pavillon de verre du Jardin botanique a fait appel également aux citoyens et à ses partenaires associatifs pour rêver aux diverses possibilités pour ce nouveau pavillon. Il offrira une salle d'expositions pour l'organisation d'événements horticoles tout en venant combler les besoins locatifs de la communauté montréalaise. Inscrite dans la continuité du Centre sur la biodiversité, des serres et de la roseraie, cette salle multifonctionnelle modulable pouvant accueillir jusqu'à 350 personnes offrira des espaces polyvalents, aérés et lumineux qui proposent une expérience unique, pour « mieux vivre la nature ».

Enfin, le dernier-né des projets de développement est un Parcours phytotechnologique qui sera réalisé au Jardin botanique sur un horizon de 10 ans : il sera composé de six pôles de démonstration de technologies basées sur les plantes (un jardin aquatique, un toit vert, un marais filtrant...) disséminés dans différentes sections du Jardin botanique.

Au Biodôme, Migration va déployer une série de parois toutes en courbes pour être utilisées comme surface de projection développant une scénographie novatrice caractérisée par l'interactivité...

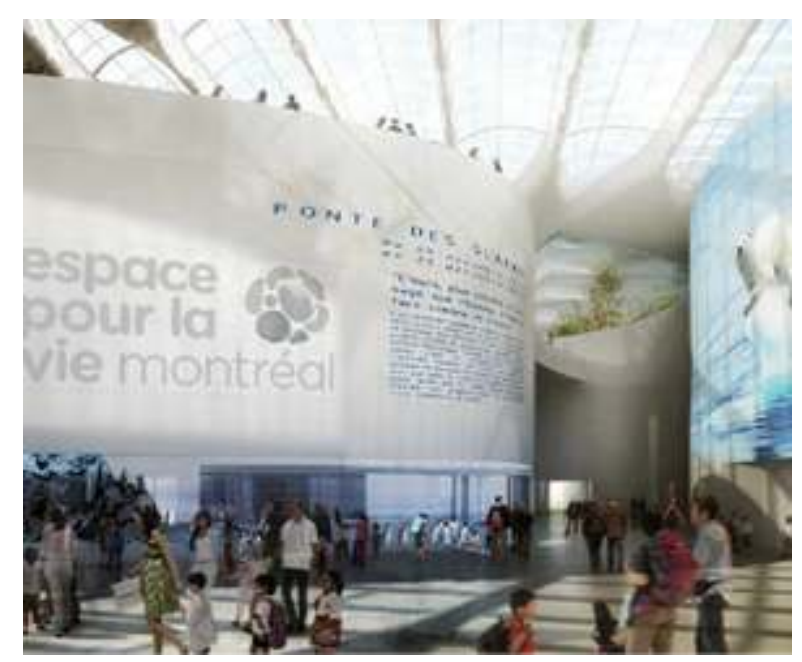

(c) Espace pour la vie 
La Métamorphose de l'Insectarium proposera un parcours inédit d'expériences immersives qui entraînera le visiteur sous terre, sous l'eau et à la surface, dans une véritable maison de l'insecte.

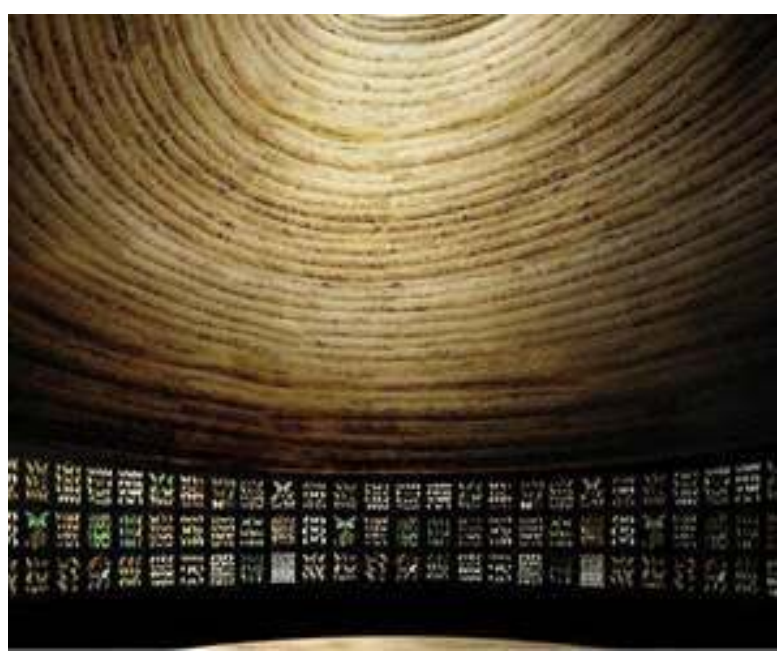

(c) Espace pour la vie

Dans la continuité du Centre sur la biodiversité, des serres et de la roseraie, le Pavillon de verre du Jardin botanique intègrera dans ses parois une roseraie sur espalier.

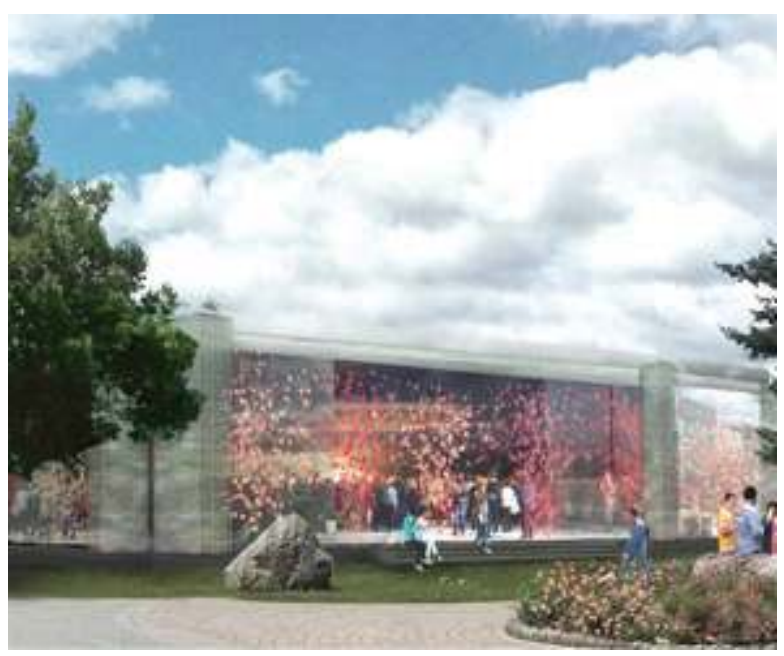

(C) Espace pour la vie

\section{NOTES}

1. Bourque, P., Landry, J. et Poussard, A. Montréal, le pari de la douceur, Écodécision, vol. 3, 1992.

2. Rahm, I. J'aime jardiner et rapporter quelque chose à la maison. Le Jardin botanique comme outil de développement des jeunes, Publication de l'ACFAS, printemps 2015.

3. Citoyenneté et biodiversité : une réflexion sur les sciences participatives, La Lettre de l'OCIM, $\mathrm{n}$ •144, novembre-décembre 2012, pp. 5-66. 
4. Janes, R.-R. Museums in a Troubled World : Irrelevance, Collapse or Renewal. Routledge, 2009.

5. Wassenberg, C.-L., Goldenberg, M.-A. \& Soule, K.-E. Benefits of botanical garden visitation : a means-end study, Urban Forestry \& Urban greening, n¹4, 2015, pp. 148-155.

6. Louv, R. Last Child in the Woods : Saving Our Children From Nature-Deficit Disorder. Algonquin Books of Chapel Hill, 2005

7. Signature officielle de la déclaration: http://espacepourlavie.ca/actualites

8. Wilson, E.-O. Biophilia. Harvard University Press, 1984.

\section{RÉSUMÉS}

Le Biodôme, l'Insectarium, le Jardin botanique et le Planétarium Rio Tinto Alcan forment ensemble un lieu où la nature et les sciences sont à l'honneur, un Espace pour la vie. C'est un mouvement participatif auquel les communautés d'ici et d'ailleurs sont invitées à participer. Et c'est aussi un engagement à protéger la biodiversité de la planète au sein d'une démarche initiée dès la naissance du Jardin botanique dans les années 1930.

\section{INDEX}

Mots-clés : participatif, citoyen, jardin botanique, insectarium, planetarium

\section{AUTEUR}

\section{JOHANNE LANDRY}

chef de division Programmes publics, Jardin botanique, Espace pour la vie Montréal

johanne.landry@ville.montreal.qc.ca 